\title{
Analysis of Obata's Differential Equations on Pointwise Semislant Warped Product Submanifolds of Complex Space Forms via Ricci Curvature
}

\author{
Amira A. Ishan (iD) \\ Department of Mathematics, College of Science, Taif University, P.O. Box 11099, Taif 21944, Saudi Arabia \\ Correspondence should be addressed to Amira A. Ishan; amiraaishan@gmail.com
}

Received 11 April 2021; Accepted 10 June 2021; Published 27 June 2021

Academic Editor: G. Muhiuddin

Copyright (c) 2021 Amira A. Ishan. This is an open access article distributed under the Creative Commons Attribution License, which permits unrestricted use, distribution, and reproduction in any medium, provided the original work is properly cited.

\begin{abstract}
The present paper studies the applications of Obata's differential equations on the Ricci curvature of the pointwise semislant warped product submanifolds. More precisely, by analyzing Obata's differential equations on pointwise semislant warped product submanifolds, we demonstrate that, under certain conditions, the base of these submanifolds is isometric to a sphere. We also look at the effects of certain differential equations on pointwise semislant warped product submanifolds and show that the base is isometric to a special type of warped product under some geometric conditions.
\end{abstract}

\section{Introduction}

The study of Obata [1] has become a vital investigation technique for geometric analysis. Basically, Obata described the Obata equation as a characterization theorem for a regular sphere in terms of a differential equation. According to Obata, if $\left(M^{n}, g\right)$ is a complete Riemannian manifold, then the nonconstant function $f$ on $M^{n}$ satisfies the differential equation $\nabla^{2} f+c f g=0$ or $\operatorname{Hessian}(f)+c f g=0$ if and only if $M^{n}$ is isometric to $n$-dimensional sphere of radius $c$. A significant number of studies have been conducted on this topic. As a result, the Euclidean space, Euclidean sphere, and complex projective space are recognized domains in the analysis of differential geometry of manifolds, for instance, [2-17]. As a special case, the differential equation $\nabla^{2} f=c g$ signifies the Euclidean space, where $c$ is a constant; infact, this was proved by Tashiro [17]. In [18], Lichnerowicz has proved that, under some geometric condition, there exists an isometry between $\left(M^{n}, g\right)$ and $S^{n}$. However, Deshmukh and Al-Solamy used Obata's differential equation and showed the connected Riemannian manifold isometric to $n$-dimensional sphere of radius $c$ if the Ricci curvature of $\left(M^{n}, g\right)$ satisfies the inequality $0<$ Ric $\leq(n-1)\left(2-\left(n c / \mu_{1}\right) c\right)$ for a constant $c$, where $\mu_{1}$ is the first eigenvalue of the Laplacian. Furthermore, Al-Dayael and Khan [19] proved that, under certain conditions, the base of contact CR-warped product submanifolds $N_{T} \times{ }_{f} N_{\perp}$ is isometric to a sphere. Recently, Mofarreh et al. [20] used Obata's differential equation on warped product submanifolds of Sasakian space form and established some characterization.

On the contrary, Bishop and O'Neill [21] evaluated the geometry of manifolds having negative curvature and noticed that Riemannian product manifolds do have nonnegative curvature. As a result, they came up with the recommendation of warped product manifolds, which are described as follows.

Consider two Riemannian manifolds $\left(L_{1}, g_{1}\right)$ and $\left(L_{2}, g_{2}\right)$ with corresponding Riemannian metrics $g_{1}$ and $g_{2}$ and $\psi: L_{1} \longrightarrow R$ as a positive differentiable function. If $x$ and $y$ are projection maps such that $x: L_{1} \times L_{2} \longrightarrow L_{1}$ and $y: L_{1} \times L_{2} \longrightarrow L_{2}$, which are defined as $x(m, n)=m$ and $y(m, n)=n \forall(m, n) \in L_{1} \times L_{2}$, then $\bar{L}=L_{1} \times L_{2}$ is called warped product manifold if the Riemannian structure on $L$ satisfies

$$
g(\bar{E}, \bar{F})=g_{1}\left(x_{*} \bar{E}, x_{*} \bar{F}\right)+(\psi \circ x)^{2} g_{2}\left(y_{*} \bar{E}, y_{*} \bar{F}\right),
$$

for all $\bar{E}, \bar{F} \in T \bar{L}$, the function $\psi$ is warping function of $L_{T}^{n_{1}} \times L_{2}$. The Riemannian product manifold is a special case 
of warped product manifold in which the warping function is constant. The study of Bishop and O' Neill [21] revealed that these types of manifolds have wide range of applications in physics and theory of relativity. It is well known that the warping function is the solution of some partial differential equations, for example, Einstein field equation can be solved by the approach of warped product [22]. The warped product is also applicable in the study of space time near to black holes [23].

$$
\bar{R}(E, F, G, H)=\frac{c}{4}[g(F, G) g(E, H)-g(E, G) g(F, H)+g(E, J G) g(J F, H)-g(F, J G) g(J E, H)+2 g(E, J F) J G]
$$

$$
R(\chi)=\sum_{\substack{\alpha=1 \\ \alpha \neq u}}^{n} \kappa\left(e_{\alpha} \wedge e_{u}\right)
$$

The submanifold $M$ of an almost Hermitian manifold $\bar{M}$ is called a pointwise slant submanifold if, at each point $x \in M$, the Wirtinger angle $\theta(X)$ between $J X$ and $T_{x} M$ is independent of the choice of the nonzero vector $X \in T_{x} M$. In this case, the angle $\theta$ is treated as a function on $M$, which is called the slant function of the pointwise slant submanifold [24].

A submanifold $M$ of an almost Hermitian manifold $\bar{M}$ is called a pointwise semislant submanifold if there exist two orthogonal complementary distributions $D$ and $D^{\perp}$ such that $T M=D \oplus D^{\perp}$, where $D$ is a holomorphic distribution, i.e., $J D=D$ and $D^{\perp}$ is a pointwise slant distribution with slant function $\theta$ [24].

Biwarped product submanifolds of the type $M=N_{T} \times{ }_{f_{1}} N_{\perp} \times{ }_{f_{2}} N_{\theta}$ of a Kaehler manifold $\bar{M}$ have been studied by Tastan [25], where $N_{T}, N_{\perp}$, and $N_{\theta}$ are invariant, anti-invariant, and slant submanifolds. Furthermore, Khan and Khan [26] extended the study of biwarped product submanifold in the complex space form; more precisely, they studied the warped product of the type $M=$ $N_{T} \times{ }_{f_{1}} N_{\perp} \times{ }_{f_{2}} N_{\theta}$, where $N_{T}, N_{\perp}$, and $N_{\theta}$ are invariant, anti-invariant, and slant submanifolds of the complex space form $\bar{M}(c)$, respectively. Recently, Ishan and Khan [27] used biwarped product submanifolds and calculated the Ricci curvature inequalities of biwarped product submanifold. Simultaneously, as a special case, they also obtained the Ricci curvature for pointwise semislant warped product submanifold of the form $M=N_{T} \times{ }_{f} N_{\theta}$, where $N_{T}$ is the invariant submanifold and $N_{\theta}$ is the pointwise slant submanifold. More details of these types of submanifolds are available in [24, 28]. Basically, Ishan and Khan [27] proved the following result.

Theorem 1 (see Corollary 4.2 in [27]). Let $M^{n}=N_{T}^{n_{1}} \times{ }_{f} N_{\theta}^{n_{2}}$ be a pointwise semislant warped product submanifold isometrically immersed in a complex space form $\bar{M}(c)$. Then, for

each orthogonal unit vector field $\chi \in T_{x} M$, either tangent to for all $E, F \in T_{x} M$, where $R$ is the Riemannian curvature tensor. The above tensor is called the Ricci tensor. If we fix a distinct vector $e_{u}$ from $\left\{e_{1}, \ldots, e_{n}\right\}$ on $M^{n}$, which is governed by $\chi$, then the Ricci curvature is defined by

\section{Preliminaries
Let $\bar{M}$ be an almost Hermitian manifold with an almost} complex structure $J$ and almost Hermitian metric $g$, i.e., $J^{2}=$ $-I$ and $g(J E, J F)=g(E, F)$, for all $E, F \in T \bar{M}$. If $J$ is parallel
with respect to the Levi-Civita connection $\bar{\nabla}$ on $\bar{M}$, i.e., $\left(\bar{\nabla}_{E} J\right) F=0$, for all $E, F \in T \bar{M}$, then $(\bar{M}, g, J)$ is called the Kaehler manifold. A Kaehler manifold $\bar{M}$ is called the
complex space form if and only if it has constant holomorphic sectional curvature denoted by $\bar{M}(c)$. The cur-

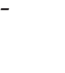

$$
\begin{aligned}
& H(x)=\frac{1}{n} \sum_{i=1}^{n} \sigma\left(e_{i}, e_{i}\right), \\
& \|H\|^{2}=\frac{1}{n^{2}} \sum_{i, j=1}^{n} g\left(\sigma\left(e_{i}, e_{i}\right), \sigma\left(e_{j}, e_{j}\right)\right),
\end{aligned}
$$


$M_{T}^{n_{1}}$ or $M_{\theta}^{n_{2}}$, the Ricci curvature satisfies the following inequalities:

(i) If $\chi$ is tangent to $M_{T}^{n_{1}}$, then

$$
\frac{1}{4} n^{2}\|H\|^{2} \geq R(\chi)+\frac{n_{2} \Delta f}{f}+\frac{c}{4}\left(n-n_{1} n_{2}-\frac{1}{2}\right) \text {. }
$$

(ii) If $\chi$ is tangent to $M_{\theta}^{n_{2}}$, then

$$
\frac{1}{4} n^{2}\|H\|^{2} \geq R(\chi)+\frac{n_{2} \Delta f}{f}+\frac{c}{4}\left(n-n_{1} n_{2}+1-\frac{3}{2} \cos ^{2} \theta\right)
$$

where $n_{1}$ and $n_{2}$ are the dimensions of the invariant submanifold $N_{T}^{n_{1}}$ and the pointwise slant submanifold, respectively.

The equality case can be seen in [27]. Moreover, for the warped product submanifold $M=M_{T}^{n_{1}} \times{ }_{f} N_{\perp}^{n_{2}}$, we have $\left(n_{2} \Delta f / f\right)=n_{2}\left(\Delta \ln f-\|\nabla \ln f\|^{2}\right)$ [8]. Using this fact in the Theorem 1, we obtain the following theorem.

Theorem 2. Let $M^{n}=N_{T}^{n_{1}} \times{ }_{f} N_{\theta}^{n_{2}}$ be a pointwise semislant warped product submanifold isometrically immersed in a complex space form $\bar{M}(c)$; then, for each orthogonal unit vector field $\chi \in T_{x} M$, either tangent to $N_{T}^{n_{1}}$ or $N_{\theta}^{n_{2}}$, the Ricci curvature satisfies the following inequalities:

(i) If $\chi$ is tangent to $N_{T}^{n_{1}}$, then

$$
R(\chi)+n_{2} \Delta \ln f \leq \frac{1}{4} n^{2}\|H\|^{2}+n_{2}\|\nabla \ln f\|^{2}-\frac{c}{4}\left(n-n_{1} n_{2}-\frac{1}{2}\right) .
$$

(ii) If $\chi$ is tangent to $\mathrm{N}_{\theta}^{n_{2}}$, then

$$
R(\chi)+n_{2} \Delta \ln f \leq \frac{1}{4} n^{2}\|H\|^{2}+n_{2}\|\nabla \ln f\|^{2}-\frac{c}{4}\left(n-n_{1} n_{2}+1-\frac{3}{2} \cos ^{2} \theta\right),
$$

where $n_{1}$ and $n_{2}$ are the dimensions of the invariant submanifold $N_{T}^{n_{1}}$ and the pointwise slant submanifold $N_{\theta}^{n_{2}}$, respectively.

\section{Main Results}

In this section, we study the application of Obata's differential equation on pointwise semislant submanifolds $M^{n}=$ $M_{T}^{n_{1}} \times_{f} M_{\theta}^{n_{2}}$ in the complex space form $\bar{M}(c)$ by using the Ricci curvature. Now, we have the following result.
Theorem 3. Let $M^{n}=N_{T}^{n_{1}} \times_{f} N_{\theta}^{n_{2}}$ be a compact orientable pointwise semislant warped product submanifold isometrically immersed in a complex space form $M^{m}(c)$ with positive Ricci curvature $R(\chi) \geq 0, \chi \in T N_{T}^{n_{1}}$, satisfying the following relation:

$$
\left\|\nabla^{2} \lambda\right\|^{2}=\frac{3 \mu_{1} c}{n_{1} n_{2}}\left(n-n_{1} n_{2}-\frac{1}{2}\right)-\frac{3 \mu_{1} n^{2}}{4 n_{1} n_{2}}\|H\|^{2},
$$

where $\mu_{1}>0$ is an eigenvalue of the warping function $\lambda=\ln f$. Then, the base manifold $N_{T}^{n_{1}}$ is isometric to the sphere $S^{n_{1}}\left(\mu_{1} / n_{1}\right)$ with constant sectional curvature $\left(\mu_{1} / n_{1}\right)$.

Proof. Let $\chi \in T N_{T}^{n_{1}}$, and consider that $\lambda=\ln f$ and define the following relation as

$$
\left\|\nabla^{2} \lambda-t \lambda I\right\|^{2}=\left\|\nabla^{2} \lambda\right\|^{2}+t^{2} \lambda^{2}\|I\|^{2}-2 t \lambda g\left(\nabla^{2} \lambda, I\right),
$$

where $I$ is the identity operator on the submanifold $T N_{T}^{n_{1}}$, and we know that $\|I\|^{2}=\operatorname{trace}\left(I I^{*}\right)=n_{1}$ and

$$
g\left(\nabla^{2} \lambda, I^{*}\right)=\operatorname{trace}\left(\nabla^{2} \lambda, I^{*}\right)=\operatorname{trace} \nabla^{2} \lambda .
$$

Then, equation (13) transforms to

$$
\left\|\nabla^{2} \lambda-t \lambda I\right\|^{2}=\left\|\nabla^{2} \lambda\right\|^{2}+n_{1} t^{2} \lambda^{2}-2 t \lambda \Delta \lambda .
$$

Assuming $\mu_{1}$ is an eigenvalue of the eigen function $\lambda$, then $\Delta \lambda=\mu_{1} \lambda$. Thus, we obtain

$$
\left\|\nabla^{2} \lambda-t \lambda I\right\|^{2}=\left\|\nabla^{2} \lambda\right\|^{2}+\left(n_{1} t^{2}-2 t \lambda\right) \lambda^{2} .
$$

On the contrary, we obtain $\Delta \lambda^{2}=2 \lambda \Delta \lambda+\|\nabla \lambda\|^{2}$ or $\mu_{1} \lambda^{2}=2 \mu_{1} \lambda^{2}+\|\nabla \lambda\|^{2}$ which implies that $\lambda^{2}=-\left(1 / \mu_{1}\right)\|\nabla \lambda\|^{2}$; using this in equation (16), we have

$$
\left\|\nabla^{2} \lambda-t \lambda I\right\|^{2}=\left\|\nabla^{2} \lambda\right\|^{2}+\left(2 t-\frac{n_{1} t^{2}}{\mu_{1}}\right)\|\nabla \lambda\|^{2} .
$$

In particular, $t=-\left(\mu_{1} / n_{1}\right)$ in equation (17), and integrating with respect to volume element $\mathrm{d} V$,

$$
\int_{M^{n}}\left\|\nabla^{2} \lambda+\frac{\mu_{1}}{n_{1}} \lambda I\right\|^{2} \mathrm{~d} V=\int_{M^{n}}\left\|\nabla^{2} \lambda\right\|^{2} \mathrm{~d} V-\frac{3 \mu_{1}}{n_{1}} \int_{M^{n}}\|\nabla \lambda\|^{2} \mathrm{~d} V .
$$

Integrating inequality (10) and using the fact $\int_{M^{n}} \Delta \phi \mathrm{d} V=0$, we have

$$
\int_{M^{n}} \operatorname{Ric}(\chi) \mathrm{d} V \leq \frac{n^{2}}{4} \int_{M^{n}}\|H\|^{2} \mathrm{~d} V+n_{2} \int_{M^{n}}\|\nabla \lambda\|^{2} \mathrm{~d} V+-\frac{c}{4}\left(n-n_{1} n_{2}-\frac{1}{2}\right) \operatorname{Vol}\left(M^{n}\right) .
$$

From equations (18) and (19), we derive

$$
\frac{1}{n_{2}} \int_{M^{n}} \operatorname{Ric}(\chi) \mathrm{d} V \leq \frac{n^{2}}{4 n_{2}} \int_{M^{n}}\|H\|^{2} \mathrm{~d} V-\frac{n_{1}}{3 \mu_{1}} \int_{M^{n}}\left\|\nabla^{2} \lambda+\frac{\mu_{1}}{n_{1}} \lambda I\right\|^{2} \mathrm{~d} V+\frac{n_{1}}{3 \mu_{1}} \int_{M^{n}}\left\|\nabla^{2} \lambda\right\|^{2} \mathrm{~d} V-\frac{c}{4}\left(n-n_{1} n_{2}-\frac{1}{2}\right) \operatorname{Vol}\left(M^{n}\right) .
$$


According to assumption $\operatorname{Ric}(\chi) \geq 0$, the above inequality gives

$$
\int_{M^{n}}\left\|\nabla^{2} \lambda+\frac{\mu_{1}}{n_{1}} \phi I\right\|^{2} \mathrm{~d} V \leq \frac{3 n^{2} \mu_{1}}{4 n_{1} n_{2}} \int_{M^{n}}\|H\|^{2} \mathrm{~d} V+\int_{M^{n}}\left\|\nabla^{2} \lambda\right\|^{2} \mathrm{~d} V-\frac{3 \mu_{1} c}{n_{1} n_{2}}\left(n-n_{1} n_{2}-\frac{1}{2}\right) \operatorname{Vol}\left(M^{n}\right) .
$$

From equation (12), we obtain

$$
\int_{M^{n}}\left\|\nabla^{2} \lambda+\frac{\mu_{1}}{n_{1}} \lambda I\right\|^{2} \mathrm{~d} V \leq 0
$$

but we know that

$$
\int_{M^{n}}\left\|\nabla^{2} \lambda+\frac{\mu_{1}}{n_{1}} \lambda I\right\|^{2} \mathrm{~d} V \geq 0
$$

Combining the last two statements, we obtain

$$
\int_{M^{n}}\left\|\nabla^{2} \lambda+\frac{\mu_{1}}{n_{1}} \lambda I\right\|^{2} \mathrm{~d} V=0 \Longrightarrow \nabla^{2} \lambda=-\frac{\mu_{1}}{n_{1}} \lambda I
$$

Since the warping function $\lambda=\ln f$ is not constant function on $M^{n}$, so equation (24) is Obata's [1] differential equation with constant $c=\left(\mu_{1} / n_{1}\right)>0$. As $\mu_{1}>0$, therefore, the base submanifold $N_{T}^{n_{1}}$ is isometric to the sphere $S^{n_{1}}\left(\mu_{1} / n_{1}\right)$ with constant sectional curvature $\left(\mu_{1} / n_{1}\right)$. This proves the theorem.

If we consider that the unit vector field $\chi \in T N_{\theta}^{n_{2}}$, then, by adopting the similar steps as in the proof of Theorem 3 , we have the following theorem.

Theorem 4. Let $M^{n}=N_{T}^{n_{1}} \times{ }_{f} N_{\theta}^{n_{2}}$ be a compact orientable pointwise semislant warped product submanifold isometrically immersed in a complex space form $M^{m}(c)$ with positive Ricci curvature $R(\chi) \geq 0, \chi \in T N_{\theta}^{n_{2}}$, satisfying the following relation:

$$
\left\|\nabla^{2} \lambda\right\|^{2}=\frac{3 \mu_{1} c}{n_{1} n_{2}}\left(n-n_{1} n_{2}+1-\frac{3}{2} \cos ^{2} \theta\right)-\frac{3 \mu_{1} n^{2}}{4 n_{1} n_{2}}\|H\|^{2},
$$

where $\mu_{1}>0$ is an eigenvalue of the warping function $\lambda=\ln f$. Then, the base manifold $N_{T}^{n_{1}}$ is isometric to the sphere $S^{n_{1}}\left(\mu_{1} / n_{1}\right)$ with constant sectional curvature $\left(\mu_{1} / n_{1}\right)$.
In [16], García-Rio et al. studied another version of Obata's differential equation in the characterization of Euclidean sphere. Basically, they proved that if $\lambda$ be a realvalued nonconstant function on a Riemannian manifold satisfying $\Delta \lambda+\mu_{1} \lambda=0$ such that $\lambda<0$, then $M^{n}$ is isometric to a warped product of the Euclidean line and a complete Riemannian manifold whose warping function $\lambda$ is the solution of the following differential equation:

$$
\frac{\mathrm{d}^{2} \lambda}{\mathrm{d} t^{2}}+\mu_{1} \lambda=0
$$

Motivated by the study of García-Rio et al. [16] and Ali et al. [2], we obtain the following characterization.

Theorem 5. Let $M^{n}=N_{T}^{n_{1}} \times{ }_{f} N_{\theta}^{n_{2}}$ be a compact orientable pointwise semislant warped product submanifold isometrically immersed in a complex space form $M^{m}(c)$ with positive Ricci curvature $R(\chi)>0, \chi \in T N_{T}^{n_{1}}$, satisfying one of the following relation:

$$
\left\|\nabla^{2} \lambda\right\|^{2}=\frac{3 \mu_{1} c}{n_{1} n_{2}}\left(n-n_{1} n_{2}-\frac{1}{2}\right)-\frac{3 \mu_{1} n^{2}}{4 n_{1} n_{2}}\|H\|^{2},
$$

where $\mu_{1}<0$ is a negative eigenvalue of the eigenfunction $\lambda=\ln \psi$. Then, $N_{T}^{n_{1}}$ is isometric to a warped product of the Euclidean line and a complete Riemannian manifold whose warping function $\lambda=\ln \psi$ satisfies the differential equation

$$
\frac{\mathrm{d}^{2} \lambda}{\mathrm{d} t^{2}}+\mu_{1} \lambda=0
$$

Proof. Since we assumed that the Ricci curvature is positive, then, by Myers's theorem, a complete Riemannian manifold with positive Ricci curvature is compact which means $M^{n}$ is compact contact CR-warped product submanifold with free boundary [29]. Then, by equation (20),

$$
\frac{1}{n_{2}} \int_{M^{n}} \operatorname{Ric}(\chi) \mathrm{d} V \leq \frac{n^{2}}{4 n_{2}} \int_{M^{n}}\|H\|^{2} \mathrm{~d} V-\frac{n_{1}}{3 \mu_{1}} \int_{M^{n}}\left\|\nabla^{2} \lambda+\frac{\mu_{1}}{n_{1}} \lambda I\right\|^{2} \mathrm{~d} V+\frac{n_{1}}{3 \mu_{1}} \int_{M^{n}}\left\|\nabla^{2} \lambda\right\|^{2} \mathrm{~d} V-\frac{c}{4 n_{2}}\left(n-n_{1} n_{2}-\frac{1}{2}\right) \operatorname{Vol}\left(M^{n}\right) .
$$

According to hypothesis Ricci curvature which is positive $\operatorname{Ric}(\chi)>0$, then we have

$$
\int_{M^{n}}\left\|\nabla^{2} \lambda+\frac{\mu_{1}}{n_{1}} \lambda I\right\|^{2} \mathrm{~d} V<\frac{3 n^{2} \mu_{1}}{4 n_{1} n_{2}} \int_{M^{n}}\|H\|^{2} \mathrm{~d} V+\int_{M^{n}}\left\|\nabla^{2} \phi\right\|^{2} \mathrm{~d} V-\frac{3 \mu_{1} c}{4 n_{1} n_{2}}\left(n-n_{1} n_{2}-\frac{1}{2}\right) \operatorname{Vol}\left(M^{n}\right)
$$


If equation (27) holds, then from last inequality, we get $\left\|\nabla^{2} \lambda+\left(\mu_{1} / n_{1}\right) \lambda I\right\|^{2}<0$, which is not possible; hence, $\left\|\nabla^{2} \lambda+\left(\mu_{1} / n_{1}\right) \lambda I\right\|^{2}=0$. Since $\mu_{1}<0$, then by result of [16], the submanifold $N_{T}^{n_{1}}$ is isometric to a warped product of the Euclidean line and a complete Riemannian manifold, where the warping function on $R$ is the solution of the differential equation (28), and this proves the theorem.

Similarly, if we consider the unit vector field $\xi \in T N_{\theta}^{n_{2}}$, then we have the following result, which can be verified as Theorem 5 .

Theorem 6. Suppose $M^{n}=N_{T}^{n_{1}} \times_{f} N_{\theta}^{n_{2}}$ be a compact orientable pointwise semislant warped product submanifold isometrically immersed in a complex space form $M^{m}(c)$ with positive Ricci curvature $R(\chi)>0, \chi \in T N_{\theta}^{n_{2}}$ and satisfying one of the following relation:

$$
\left\|\nabla^{2} \lambda\right\|^{2}=\frac{3 \mu_{1} c}{n_{1} n_{2}}\left(n-n_{1} n_{2}+1-\frac{3}{2} \cos ^{2} \theta\right)-\frac{3 \mu_{1} n^{2}}{4 n_{1} n_{2}}\|H\|^{2},
$$

where $\mu_{1}<0$ is a negative eigenvalue of the eigen function $\lambda=\ln \psi$. Then, $N_{T}^{n_{1}}$ is isometric to a warped product of the Euclidean line and a complete Riemannian manifold whose warping function $\lambda=\ln \psi$ satisfies the differential equation

$$
\frac{\mathrm{d}^{2} \lambda}{\mathrm{d} t^{2}}+\mu_{1} \lambda=0
$$

\section{Conclusions}

This paper studies the geometric behavior of ordinary differential equations on the pointwise semislant warped product submanifolds. More precisely, we obtain characterizing theorems for pointwise semislant warped product submanifolds of complex space forms via differential and integral theory on Riemannian manifolds. Therefore, the present study provides a wonderful correlation of theory of differential equations with the warped product submanifolds.

\section{Data Availability}

No data were used to support the findings of the study.

\section{Conflicts of Interest}

The author declares that she has no conflicts of interest.

\section{Acknowledgments}

This work was supported by Taif University Researchers Supporting Project (No. TURSP-2020/223), Taif University, Taif, Saudi Arabia.

\section{References}

[1] M. Obata, "Certain conditions for a Riemannian manifold to be isometric with a sphere," Journal of the Mathematical Society of Japan, vol. 62, pp. 333-340, 1962.
[2] A. Ali, P. Laurian-Ioan, and A. H. Alkhaldi, "Ricci curvature on warped product submanifolds in spheres with geometric applications," Journal of Geometry and Physics, vol. 146, Article ID 103510, 2019.

[3] H. Alodan, S. Deshmukh, N. B. Turki, and G.-E. Vîlcu, "Hypersurfaces of a Sasakian manifold," Mathematics, vol. 8, no. 6 , p. 877, 2020.

[4] A. Barros, J. N. Gomes, and E. Ribeiro, "A note on rigidity of the almost Ricci soliton," Archiv der Mathematik, vol. 100, no. 5, pp. 481-490, 2013.

[5] B.-Y. Chen, "A general inequality for submanifolds in complex-space-forms and its applications," Archiv der Mathematik, vol. 67, no. 6, pp. 519-528, 1996.

[6] B.-Y. Chen, "Mean curvature and shape operator of isometric immersions in real-space-forms," Glasgow Mathematical Journal, vol. 38, no. 1, pp. 87-97, 1996.

[7] M. Jamali and M. H. Shahid, "Application of Bochner formula to generalized sasakian space forms," Afrika Matematika, vol. 29, no. 7-8, pp. 1135-1139, 2018.

[8] B.-Y. Chen, "Relations between Ricci curvature and shape operator for submanifolds with arbitrary codimensions," Glasgow Mathematical Journal, vol. 41, no. 1, pp. 33-41, 1999.

[9] B.-Y. Chen, "On Ricci curvature of isotropic and Lagrangian submanifolds in complex space forms," Archiv der Mathematik, vol. 74, no. 2, pp. 154-160, 2000.

[10] S. Deshmukh and A. Al-Eid, "Curvature bounds for the spectrum of a compact Riemannian manifold of constant scalar curvature," Journal of Geometric Analysis, vol. 15, no. 4, pp. 589-606, 2005.

[11] S. Deshmukh, "Conformal vector fields and eigenvectors of laplacian operator," Mathematical Physics, Analysis and Geometry, vol. 15, no. 2, pp. 163-172, 2012.

[12] S. Deshmukh and F. Al-Solamy, "A note on conformal vector fields on a Riemannian manifold," Colloquium Mathematicum, vol. 136, no. 1, pp. 65-73, 2014.

[13] S. Deshmukh, "Characterizing spheres and Euclidean spaces by conformal vector fields," Annali di Matematica Pura ed Applicata (1923 -), vol. 196, no. 6, pp. 2135-2145, 2017.

[14] S. Deshmukh, "Almost Ricci solitons isometric to spheres," International Journal of Geometric Methods in Modern Physics, vol. 16, no. 5, Article ID 1950073, 2019.

[15] F. Erkekoglu, E. Garcia-Rio, D. N. Kupeli, and B. Unal, "Characterizing specific Riemannian manifolds by differential equations," Acta Applicandae Mathematicae, vol. 76, no. 2, pp. 195-219, 2003.

[16] E. García-Río, D. N. Kupeli, and B. Ünal, "On a differential equation characterizing Euclidean spheres," Journal of Differential Equations, vol. 194, no. 2, pp. 287-299, 2003.

[17] Y. Tashiro, "Complete Riemannian manifolds and some vector fields," Transactions of the American Mathematical Society, vol. 117, p. 251, 1965.

[18] A. Lichnerowicz, Geometrie des Groupes de Transformations, Dunod, Paris, France, 1958.

[19] I. Al-Dayael and M. A. Khan, "Ricci curvature of contact CRwarped product submanifolds in generalized Sasakian space forms admitting nearly Sasakian structure," AIMS Mathematics, vol. 6, no. 3, pp. 2132-2151, 2021.

[20] F. Mofarreh, A. Ali, N. Alluhaibi, and O. Belova, "Ricci curvature for warped product submanifolds of Sasakian space forms and its applications to differential equations," Journal of Mathematics, vol. 2021, Article ID 1207646, 15 pages, 2021.

[21] R. L. Bishop and B. O’Neill, "Manifolds of negative curvature," Transactions of the American Mathematical Society, vol. 145, pp. 1-49, 1969. 
[22] M. Berger, "Les varietes riemanniennes (1/4)-pinces," Annali della Scuola Normale Superiore di Pisa, Classe di Scienze, vol. 14, no. 4, pp. 161-170, 1960.

[23] J. K. Beem, P. Ehrlich, and T. G. Powell, Warped Product Manifolds in Relativity Selected Studies, North-Holland, Amsterdam, NY, USA, 1982.

[24] B. Sahin, "Warped product pointwise semi-slant submanifolds of Kaehler manifolds," Portugaliae Mathematica, vol. 70, no. 3, pp. 251-268, 2013.

[25] H. M. Tastan, "Biwarped product submanifolds of a Kaehler manifold," Filomat, vol. 32, no. 7, pp. 2349-2365, 2018.

[26] M. A. Khan and K. Khan, "Biwarped product submanifolds of complex space forms," International Journal of Geometric Methods in Modern Physics, vol. 16, no. 5, Article ID 1950072, 2019.

[27] A. A. Ishan and M. A. Khan, "Chen-Ricci inequality for biwarped product submanifolds in complex space forms," AIMS Mathematics, vol. 6, no. 5, pp. 5256-5274, 2021.

[28] M. A. Khan, "Warped product pointwise semi-slant submanifolds of the complex space forms," Rendiconti del Circolo Matematico di Palermo Series 2, vol. 69, no. 1, pp. 195-207, 2020.

[29] S. B. Myers, "Riemannian manifolds with positive mean curvature," Duke Mathematical Journal, vol. 8, no. 2, Article ID 401404, 1941. 